\section{$1 \mathrm{P325}$}

The effect of active peptide mobility and conformation on cell morphology

Teruaki YOSHIDA', Takuya NODZUMI ${ }^{2}$, Daisuke AKIEDA ${ }^{2}$, Satoshi SIMAMOTO $^{2}$, Tomoyasu AIZAWA ${ }^{1,2}$, Keiichi KAWANO ${ }^{2}$, Makoto DEMURA ${ }^{1}$

${ }^{i}$ Grad. Sch of LifeSci., Hokkaodo Univ., ${ }^{2}$ Grad. Sch of Sci., Hokkaodo Univ.

Laminins are main component of extracellular matrix. Laminin biological activity for cell adhesion, migration, differentiation are extensively known. By screening synthetic peptide from laminin, the minimum active sequence site was identified. Most of these peptides bind cell surface receptors, and play a critical role in a peptide-specific and cell-type specific manner. Also, by conjugating these active peptides onto solid surface, these peptides hold their activities. It is thought that these materials have a potential ability to serve as bioadhesive materials for tissue engineering. Generally, peptide mobility/conformation as well as amino acid sequence is important for binding of the peptide to its receptor. In this study, we particularly focused on peptide mobility/conformation and investigated methods for presentation of peptide from solid surface. The relationship between peptide structure and activity was examined. To determine the effect of peptide mobility on cellular interactions, spacer group was introduced to YIGSR peptide. YIGSR derived from laminin have been shown to promote nerve growth. This peptide known to have helix like conformation. By increasing the spacer group length, the peptide would have increased conformational degrees. Then conformation of YIGSR peptides was measured by solution NMR spectroscopy.Morphology of PC12 cells on these factors was measured by neurite outgrowth assay. Moreover, in order to maintain the structural flexibility of YIGSR peptide, we are investigating introduce the peptide to protein.

\section{$1 P 327$}

Mechanical force-dependent zyxin accumulation leads to actin polymerization at focal adhesions.

OHiroaki Hirata ${ }^{1,2}$, Hitoshi Tatsumi ${ }^{3}$, Masahiro Sokabe ${ }^{1,2,3}$

'ICORP/SORST, Cell Mechanosensing Project, Japan Science and Technology Agency, ${ }^{2}$ Dept. of Molecular Physiology, National Institute for Physiological Sciences, ${ }^{3}$ Dept. of Physiology, Graduate School of Medicine, Nagoya Univ.

Cell-to-substratum adhesion realized by focal adhesions (FAs) controls cellgrowth, -proliferation and -apoptosis. Therefore, molecular understanding of FAsize regulation is a critically important theme in cell biology. It has been well established that intra- and extracellular mechanical environments affect the FA-size, however, little is known about the mechanism underlying this phenomenon. FA is a site where actin polymerizes while depolymerization of actin reduces FA-size. Therefore, certain actin-modulating proteins at FA should be involved in the mechanical regulation of FA-size. In the present study, we focused on zyxin, an FA-associated protein that has actin polymerizing capability, as a candidate molecule and examined its distribution in response to changes in the mechanical environment in cultured fibloblasts. When the cell contractility was inhibited by the myosin II inhibitor, blebbistatin, clusters of zyxin disappeared from FAs, which was the earliest remarkable event we could notice. This result suggested that the zyxin assembly/disassembly is regulated by the forces applied to FAs. To confirm this idea we applied uniaxial 50\%-stretch to the blebbistatin-treated cells and found that clusters of zyxin were assembled at FAs. When Alexa568-actin was introduced into the permeabilized cell, G-actin was incorporated at zyxin-positive FAs but not at zyxin-negative ones. These results suggest that zyxin is involved in a certain mechano-responsive machinery and regulates actin polymerization at FAs in a force-dependent manner.

\section{$1 P 326$}

Structural analysis of uro-adherence factor A, UafA, from Staphylococcus saprophyticus

OEriko Matsuoka', Yoshikazu Tanaka', Makoto Kuroda², Min Yao', Nobuhisa Watanabe ${ }^{1}$, Toshiko Ohta ${ }^{2}$, Isao Tanaka ${ }^{1}$

${ }^{1}$ Faculty of Advanced Life Science, Hokkaido Univ., ${ }^{2}$ Dept. of Microbiology, Grad. School of Comprehensive Human Science, Univ. of Tsukuba

Most bacterial infections are initiated by the adherence of microorganisms to host tissues. The adherence process involves the interaction of cell wall-anchored protein adhesins with host components. These cell wall-anchored proteins usually consist of several common domains; the signal sequence, the ligand-binding domains, the unique tandem repeats, the membrane-spanning domain, and an LPXTG motif.Staphylococcus saprophyticus is a primary Gram-positive uropathogen isolated from young female outpatients presenting uncomplicated urinary tract infections (UTI). The whole genome analysis of $S$. saprophyticus was completed in 2005, and it was revealed that $S$. saprophyticus has only a specific adhesin UafA (uro-adherence factor A) which shows positive hemagglutination activity and adherence activity to human uroepithelial cells, although other staphylococci of which genome sequencing analyses have been finished have more than 10 adhesins. Such unique adhesin UafA suggests a significant contribution to causing UTI by $S$. saprophyticus. To understand the adherence process by $S$. saprophyticus, we investigated the ligand-binding domain of UafA from structural viewpoints. The initial electron density map of core structure of the ligand-binding domain has been obtained using Se-SAD phasing. The phase improvement and map interpretation are under way. In this conference, we will discuss the structural results.

\section{$1 \mathrm{P328}$}

Molecular mechanism of stretch-induced deadhesion of integrin in endothelial cells: involvement of clathrin-dependent endocytosis.

$\bigcirc$ Daisuke Kiyoshima ${ }^{1}$, Keisuke Kawakami ${ }^{2}$, Kimihide Hayakawa ${ }^{3}$, Hitoshi Tatsumi ${ }^{1}$, Masahiro Sokabe ${ }^{1,3,4}$

${ }^{1}$ Dept. Physiol., Grad. Sch. Med., Univ. Nagoya, Nagoya, Japan, ${ }^{2}$ Dept. Phys. Ther., Sch. Health Sci., Univ. Nagoya, Nagoya, Japan, ${ }^{3}$ SORST Cell-Mechanosensing Project, JST, Nagoya, Japan, ${ }^{4}$ Dept. of Molecular Physiology, NIPS. NINS, Okazaki, Japan

When subjected to uniaxial cyclic stretch, cultured human umbilical vein endothelial cells (HUVECs) gradually change their morphology towards a spindlelike shape with their major axis perpendicular to the stretch axis. For this shape remodeling, deadhesion of integrin, which is a major component of focal contact (FC), at the cell margins in the stretch axis is indispensable. To explore the molecular mechanism underlying the mechano-induced deadhesion of integrin, we developed a method by which we can apply mechanical stretch to the integrin clusters at FCs while observing the dynamics of integrin molecules that were stained with Alexa-conjugated antibody against an extracellular domain of $\beta 1$ integrin. When placed a fibronectin-coated glass bead on the apical cell surface, FCs were formed underneath the beads and connected to a particular group of basal FCs via actin stress-fibers. Mechanical stretch was applied to the basal FCs by displacing the glass bead with a glass pipette in a lateral direction. Within a minute after a step displacement of the glass bead, integrin clusters at particular basal FCs were disappeared. We observed stretch-dependent increases in the colocalization of integrin and clathrin molecules, and in the rate of endocytosis that was monitored with FM4-64, a fluorescent endocytosis marker. These results suggest that mechanical stimuli to basal FCs via actin stress fibers facilitate clathrin-dependent endocytosis of integrin. clusters at the FCs, leading to a deadhesion of the corresponding cell margin. 